\title{
MCAO for the E-ELT: preliminary design overview of the MAORY module
}

Italo Foppiani ${ }^{1, \text { a }}$, Emiliano Diolaiti ${ }^{2}$, Matteo Lombini ${ }^{2}$, Andrea Baruffolo ${ }^{3}$, Valdemaro Biliotti ${ }^{4}$, Giovanni Bregoli ${ }^{2}$, Giuseppe Cosentino ${ }^{1}$, Bernard Delabre ${ }^{5}$, Enrico Marchetti ${ }^{5}$, Laura Schreiber ${ }^{1}$, Jean-Marc Conan ${ }^{6}$, Sandro D'Odorico ${ }^{5}$, and Norbert Hubin ${ }^{5}$

${ }^{1}$ Università di Bologna Dipartimento di Astronomia, via Ranzani 1, 40127 Bologna, Italy

2 INAF - Osservatorio Astronomico di Bologna, via Ranzani 1, 40127 Bologna, Italy

3 INAF - Osservatorio Astronomico di Padova, vicolo dell'Osservatorio 5, 35122 Padova, Italy

4 INAF - Osservatorio Astrofisico di Arcetri, largo E. Fermi 5, 50125 Firenze, Italy

5 ESO European Organisation for Astronomical Research in the Southern Hemisphere, Karl-Schwarzschild-Str. 2, 85748 Garching bei München, Germany

${ }^{6}$ ONERA Office National d'Etudes et de Recherches Aerospatiales, B.P. 72, 92322 Châtillon, France

\begin{abstract}
MAORY is the Multi-conjugate Adaptive Optics RelaY module for the European Extremely Large Telescope. It will be located on the Nasmyth platform of the telescope to feed scientific instruments. The module is supposed to re-image one to one the telescope focal plane with diffraction limited optical quality and to provide multi-conjugate adaptive optics correction of the wavefront distortion induced by the atmosphere. The system is based on six laser guide stars for sensing the wavefront distortion and three deformable mirrors for correcting it. A dichroic is used to split the laser light from the light of the scientific and natural guide stars channel. Two output ports, selected by the dichroic orientation, are foreseen: one in a gravity invariant configuration and one in a vertical position to feed large instruments. In this article the current optical and mechanical design are presented together with the thermal analysis.
\end{abstract}

\section{Introduction}

As well known from literature [1] the multi conjugated Adaptive Optics (AO) concept is based on several Deformable Mirrors (DMs) conjugated at different altitudes in atmosphere that correct the wavefront distortions induced by different turbulent layers. In this way the corrected field of view (FoV) is wider than the small isoplanatic patch of few arcsecond typically achievable at visible and nearinfrared wavelengths by means of classical single conjugated AO systems [2]. MAORY is requested to provide corrected images over a $\mathrm{FoV}$ up to 2 arcminutes diameter at near-infrared wavelengths from I to $\mathrm{H}$ band and to operate at zenith angles up to $60^{\circ}$ [3] [4]. A detailed analysis and simulation [5] shows that the optimal number of DMs in this case is three. The optical system provides two intermediate pupil images which allow to locate two post focal DMs while the third DM used is the telescope mirror M4 which is deformable and conjugated to the ground.

The tomographical measurement of wavefront distortions is obtained by means of six Laser Guide Stars (LGSs) based on the atmospheric sodium layer and three Natural Guide Stars (NGSs). The NGs are needed to recover the low order modes tomography which is affected by the Tip-Tilt indetermination of the laser beacons [6]. A dichroic beam splitter reflects the light of wavelengths longer than $600 \mathrm{~nm}$, which is used by scientific instruments, while it transmits shorter wavelengths light which is used by the LGS Wavefront Sensor (WS). As baseline the LGS beacons are launched with a constellation geometry which remains fixed with respect to the telescope pupil in order to minimize the need for parameters updating during real time operations.

The two output ports of the module are selected by the dichroic orientation. In one case the beam reflected by the dichroic is parallel to the MAORY bench and reaches the vertical output port located at one edge of the bench in order to feed large and/or heavy instruments positioned beside MAORY.

\footnotetext{
a e-mail: italo.foppiani@unibo.it
} the original work is properly cited. 
In the other case the beam is reflected perpendicularly to the bench to feed a gravity invariant port underneath MAORY.

The thermal background contribution of the module optics is expected to be of the same order as the telescope one.

The expected performance of MAORY goes from about $10 \%$ to $50 \%$ Strehl ratio from $\mathrm{J}$ to $\mathrm{K}$ band as mean values on the whole 2 arcmin FoV assuming a 0.8 arcsec seeing. The expected uniformity is about $10 \%$ RMS on the whole field. Performance on smaller fields are better: up to about $60 \%$ Strehl ratio with $1 \%$ RMS variation on a $20 \operatorname{arcsec} \mathrm{FoV}$ in $\mathrm{K}$ band. Futhermore also the performance on a wider FoV is interesting: up to about $40 \%$ Strehl ratio on $2.5 \mathrm{arcmin}$ FoV in $\mathrm{K}$ band. In I band the performance is more seeing dependent than in the other bands but for a good seeing of $0.6 \operatorname{arcsec}$ the system is expected to achieve a mean Strehl ratio of about $7 \%$ on a FoV of $20 \operatorname{arcsec}$ with $10 \%$ RMS variation.

\section{Optical design}

The optical system of MAORY is based on four mirrors (M7, M9, M11, M13) with optical power which are almost off axis parabolas of diameters between about $920 \mathrm{~mm}$ and $1100 \mathrm{~mm}$. In figure 1 two views of the optical system are shown together with the basic parameters of the optical surfaces.

Top View

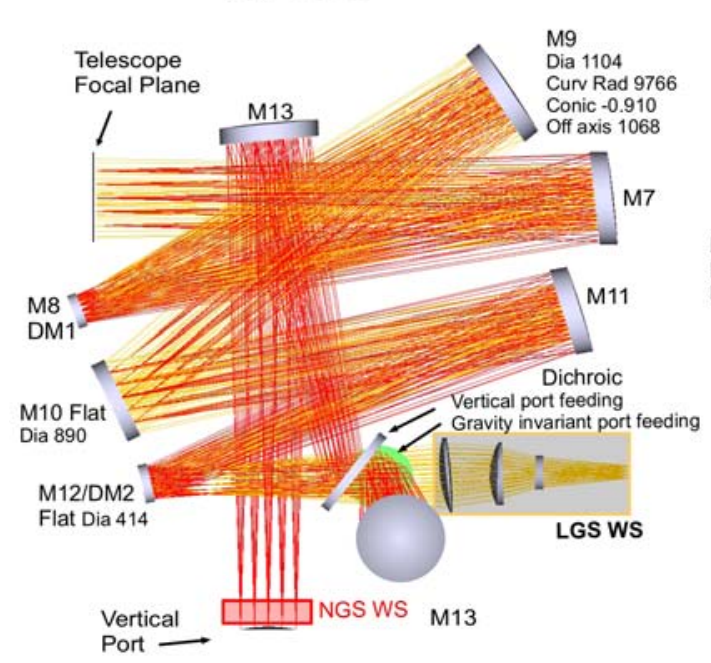

Front View

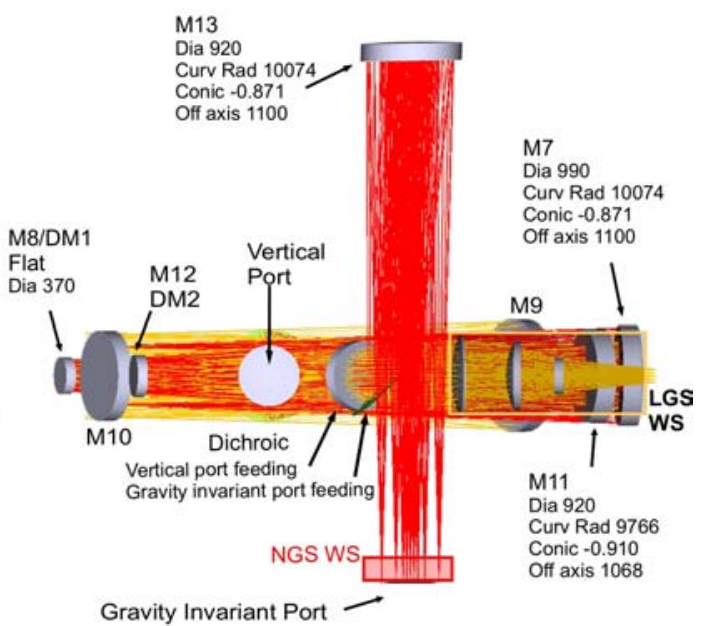

Fig. 1. MAORY Optical system

Two intermediate pupil image planes conjugated at about $13 \mathrm{Km}$ and $4 \mathrm{Km}$ of distance from the telescope allow to locate two flat deformable mirrors (M8/DM1 and M12/DM2 respectively). The DM conjugation distance is kept fixed with the zenith angle because a detailed performance analysis, carried out by means of Fourier simulation code [7], showed that the change of the relative distance of the atmospheric layers along the line of sight can be taken into account in the reconstruction algorithm without losing system performance and without changing the DM conjugation. The DMs actuator pitch is about $7 \mathrm{~mm}$, which roughly correspond to $1 \mathrm{~m}$ at the conjugated layers. An additional flat mirror (M10) is used to fold and compact the system as much as possible. The dichroic, which is about $940 \mathrm{~mm} \times 640 \mathrm{~mm}$, is located close to a pupil image in order to reduce its dimension as much as 
possible. This location requires to duplicate the last optical elements (M13) while the two output ports design requires to duplicate the NGS WS which is located close to the output port to minimize the non-common path aberrations.

The LGSs WS is based on an objective that produces a real image of the LGSs and on separate probes, one per LGS, which pick up each single LGS image and measure the wavefront by mean of a Shack-Hartmann WS. The re-imaging optics of each probe are optimized to compensate the aberrations induced by the previous optics because of the finite conjugation of the LGSs. A jitter compensation mirror and a pupil stabilization mirror are foreseen for each probe. The objective is designed in order to limit the shift of the LGSs image plane for different zenith angles, due to different distance of the sodium layer, to approximately $250 \mathrm{~mm}$. Such limited travel allows to mechanically shift all the components after the objective to keep the entrance focal plane of each probe on the LGSs images. This prevents the WS saturation due to the defocus signal.

As baseline three infrared ( $\mathrm{H}$ band) NGSs are used to recover the low order modes tomography measuring the Tip-Tilt on the wavefront of these stars. The search area of the NGSs is an annular FoV going from a diameter of 1arcminute to 2.6arcminute: the inner 1arminute FoV is kept unvignetted for imaging application while the AO correction on the outer ring is high enough to substantially increase the NGS WS performance with respect to seeing limited imaging of the NGS. In this way a good sky coverage is expected even at the galactic pole (up to 50\%). Moreover at least one NGS is used to measure the wavefront defocus because the LGSs WS measurement is affected by sodium layer altitude variation. The eventual strategy to address this issue is still under investigation since it is a delicate trade off between performance (reliability of the measurement) and sky coverage (number and flux of the required NGSs to measure the defocus term).

The two output ports (identical to each other) transmit a FoV up to 2.6arcminutes and the focal planes are characterized by a F/17.7 and a radius of curvature of about $1400 \mathrm{~mm}$. The pupil image is about $43000 \mathrm{~mm}$ before the focal plane and the nominal image quality due to the MAORY optics is about $25 \mathrm{~nm}$ of RMS wavefront error averaged across the FoV. The maximum geometrical distortion is $0.005 \%$ on the whole FoV. The expected throughput goes from about $80 \%$ to more than $90 \%$ from $0.8 \mu \mathrm{m}$ to $2.4 \mu \mathrm{m}$, assuming that a multilayer silver cooting (protected silver) is used (see Sect. 4).

\section{Mechanical design}

The mechanical design of the module is shown in figure 2.

The optical alignment specifications depend on the element considered but can be summarized in few tenth of millimetre for the positioning and from few hundreds to few tenth of milliradian for the tilt. The gravity invariant operation of the module greatly simplifies the achievement of these specifications without requiring an overwhelming mass budget: the mass of each mirror (except DMs) is estimated to be below $200 \mathrm{Kg}$ and the required mass for each mount is estimated to be similar.

The structure so far studied is based on a steel bench because it guarantees the required stiffness and because its manufacturing is quite standard for both dimensions and accuracy. The thermal expansion of such a structure, the dimension of which is about $7 \mathrm{~m} \times 7 \mathrm{~m}$, does not affect the optical performance of the system provided that it is isotropic and it is compensated by means of some refocusing. This requires that the temperature of the module is kept homogeneous within $\pm 1^{\circ} \mathrm{C}$ in order to avoid differential expansion but it does not requires to keep the temperature fixed. Another solution based on a carbon fibre bench is being evaluated in order to reduce the total weight. Unfortunately the number of the steel insertions, needed to support the mirrors, the bearing of the de-rotator of the gravity invariant port and the connection plates to divide the bench into subunits, wastes a substantial fraction the weight saving. For this reason, the increasing in cost and in production complexity of the carbon fibre technology with respect to steel technology seems not very advantageous for the bench realization. Anyway carbon fibre could be very useful to lighten the structure that supports the mirror M13 needed to feed the gravity invariant port.

The two output ports of MAORY are characterized by the same back focal distance of about $0.5 \mathrm{~m}$. The only difference (other than the orientation with respect to the gravity) is that on the gravity invariant port a mechanical de-rotator is provided to support an instrument up to 5t weight. The NGS WS 


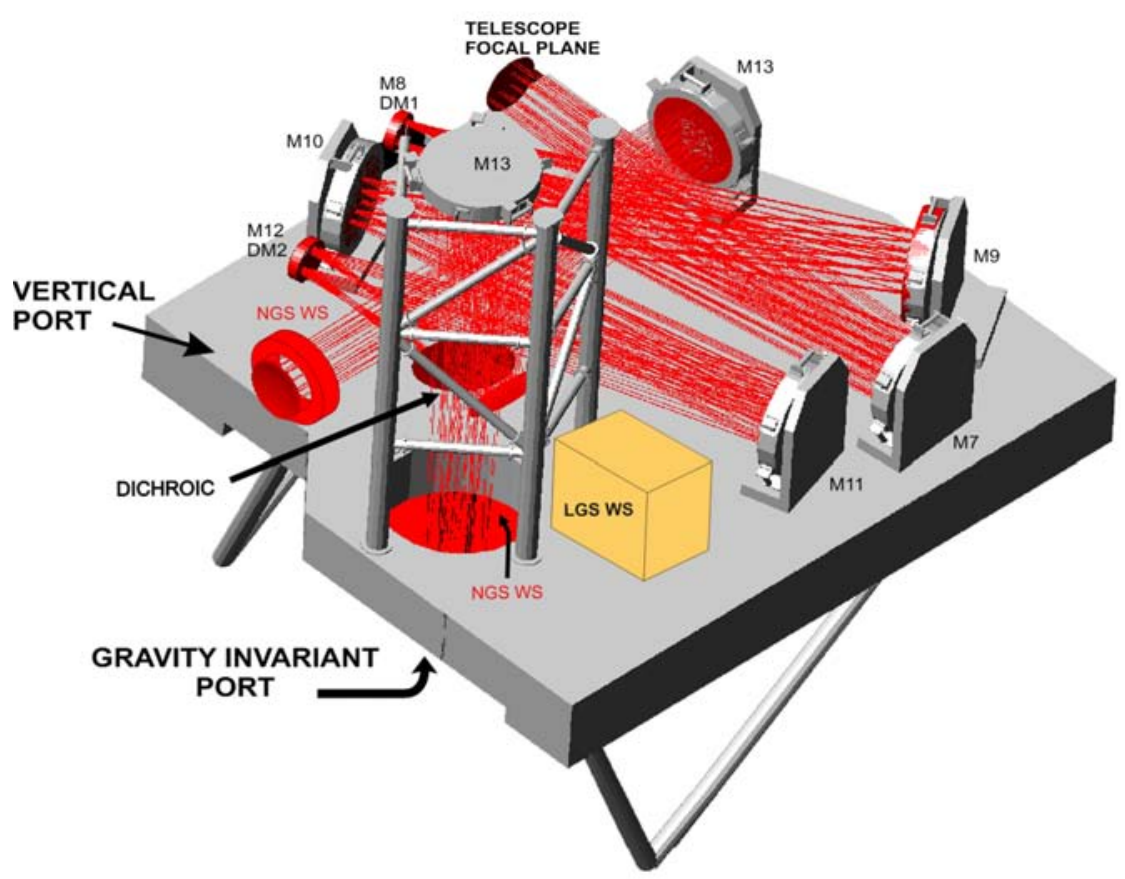

Fig. 2. MAORY mechanical layout

of this port will be mounted on the same de-rotator in order to minimize the non common path aberrations with respect to the scientific focal plane. The NGS WS on the vertical port will be mounted on a smaller dedicated de-rotator. Finally three de-rotators with a travel limited to $\pm 30^{\circ}$ are foreseen inside MAORY: two for the post-focal DMs and one for the probes of the LGS WS. The aim of this three derotators together with the LGS projection geometry, which is fixed with respect to the telescope pupil, is to keep the orientation of the DMs and the LGS WS fixed with respect to each other. This scheme minimizes calibration issues and the need for parameters updating during real time operations. In this way the AO control parameters, several tens of GB of data, need to be updated every few minutes. A scheme without post focal DMs de-rotation and LGS projection fixed with respect to the sky requires to update $\mathrm{AO}$ parameters several times per second that appears a very challenging task also for future technology. Nevertheless the current scheme is the most complex from the mechanical point of view but its study does not preclude or affect a different eventual scheme. The mechanical layout would be basically the same even without de-rotation of the post focal DMs.

A cover based on aluminium panels is foreseen to protect the optics and to control the radiation exchange with the ambient. In this way the bench temperature can be kept as uniform as required. A kind of hexapod (i.e. three supports with a V shape) attached to the Nasmyth platform is foreseen to support the whole structure. The total weight is expected to range from about $15 \mathrm{t}$ to $20 \mathrm{t}$ depending on the whether a chilling system is implemented.

\section{Thermal analysis}

The thermal emission of the MAORY optics has been studied in order to evaluate its contribution to the total background radiation up to $2.5 \mu \mathrm{m}$ wavelengths.

The total emissivity of the module optics has been calculated using the Kirchhoff's law and assuming that all the optical elements, seven mirrors and one dichroic, irradiate as grey bodies. A reflectivity of the mirrors as high as $99 \%$ has been considered assuming that a multilayer silver coating (protected silver) is used [9]. A reflectivity of $0.98 \%$ above $2 \mu \mathrm{m}$ has been assumed for the dichroic. 
The thermal contribution of MAORY optics to the observational background has been evaluated for both imaging and spectroscopic applications.

In the case of imaging the $\mathrm{K}_{\mathrm{S}}$ band has been taken as reference since this is the longest wavelength band of MICADO, the high resolution imager for the E-ELT [8], that is a possible client instrument for the gravity invariant port. The total emission has been calculated integrating over the wavelengths the product of the emissivity by the Planck function by the filter transmission curve. In order to evaluate its impact on the observation condition the sky background model and the telescope emission suggested by ESO have been used (http : //www.eso.org/sci/facilities/eelt/science/drm/tech_data/). In figure 3 the thermal emission of MAORY and of the telescope are shown normalized with respect to the sky emission. Both telescope and MAORY are supposed to be at the same ambient temperature. The dashed lines represent the requirement of MAORY not to exceed the $50 \%$ of the sum of sky and telescope emission and the goal of $10 \%$.

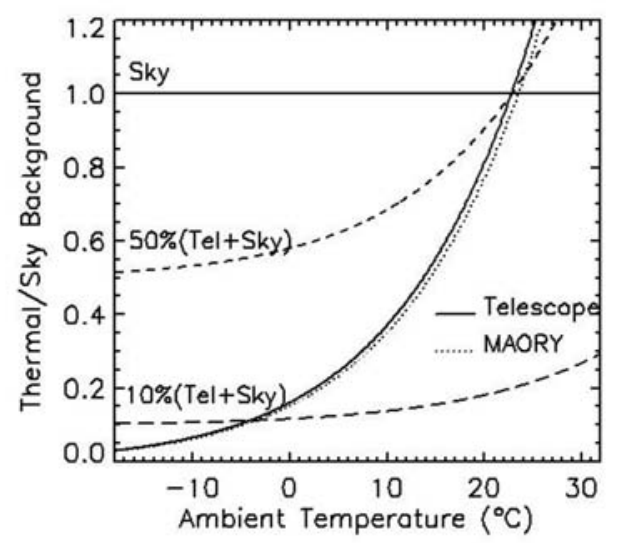

Fig. 3. MAORY thermal emission in $\mathrm{K}_{\mathrm{S}}$ band. The dashed lines represent the $10 \%$ and $50 \%$ limits of the summed sky plus telescope background.

As clear from figure 3 the MAORY emission does not exceed the $50 \%$ limit for an ambient temperature up to about $25^{\circ} \mathrm{C}$ which is very unlikely to be achieved during night time observation. From the same figure it is clear that a moderate chilling below $0^{\circ} \mathrm{C}$ is needed to reach the $10 \%$ goal.

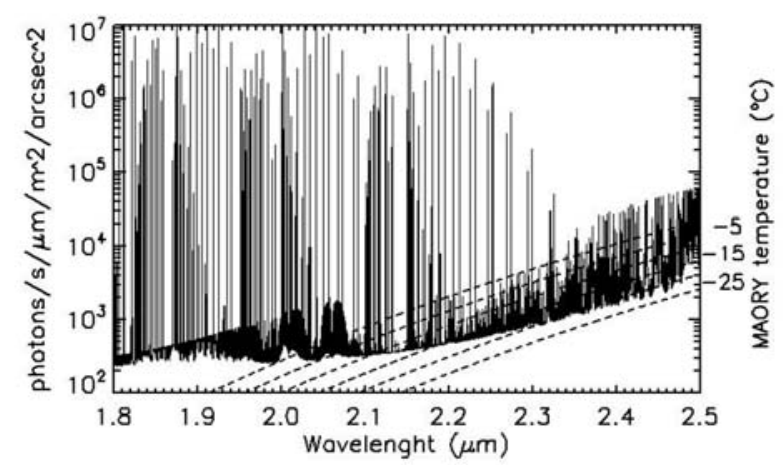

Fig. 4. Estimated $10 \%$ level of the background due to sky and telescope; the dashed lines represent the emission of the MAORY optics at different temperatures 
The case of spectroscopic applications is for sure the most demanding since the sky background continuum between the emission lines of the hydroxyl molecule $(\mathrm{OH})$ can be very low. The same telescope emission and sky model have been used to estimate the spectroscopic background up to $2.5 \mu \mathrm{m}$. In figure 4 the limit that corresponds to the $10 \%$ of the estimated background level given by the sum of sky and telescope contribution is shown from $1.8 \mu \mathrm{m}$ to $2.5 \mu \mathrm{m}$. An ambient temperature of $0^{\circ} \mathrm{C}$ is assumed. The dashed lines represent the emission of the MAORY optics assuming they are chilled at different temperature. It is clear that in order not to exceed the $10 \%$ of the sky plus telescope emission the module must be chilled to about $-20^{\circ} \mathrm{C}$. Considering a $50 \%$ limit the chilling temperature would be about $-5^{\circ} \mathrm{C}$.

\section{Conclusion}

The preliminary design of MAORY is still in progress since this phase A study is foreseen to finish by the end of this year (2009). The optical design so far developed is likely to be close to the final version while the mechanical design is expected to be refined to allow more clearance and more clean interface surfaces for client instruments.

From thermal analysis it is clear that for imaging application there is no need to chill the system, at least to fulfill the requirement not to exceed $50 \%$ of the summed background due to the telescope and to the sky. A chilling system able to reach a temperature of about $-20^{\circ} \mathrm{C}$ is anyway under study in order not to compromise spectroscopic applications and to reach the goal for imaging. Unfortunately the increasing in weight due to the chilling system is estimated to be about $30 \%$ that could be a serious overload problem for the telescope Nasmith platform. The eventual implementation of the chilling system will depend on a delicate trade off among mass budget, instrument cost and scientific applications.

\section{Acknowledgements}

This work was supported by the European Community (Framework Programme 6, ELT Design Study, contract No 011863; Framework Programme 7, Preparing for the Construction of the European Extremely Large Telescope, contract No INFRA-2007-2.2.1.28) and by the European Southern Observatory (Agreement No 16669/ESO/INS/07/17243/LCO).

\section{References}

1. Beckers J. M., Annu. Rev. Astron. Astrophys. 31, (1993) page 13-62

2. Rigaut F. J., Ellerbroek B. L., Flicker R., Proc. SPIE 4007, (2000) page 1022-1031

3. Hubin N., Review of AO systems studied for the E-ELT, this Symposium.

4. Diolaiti E., Conan J-M., Foppiani I., Lombini M., Marchetti E., Petit C., Robert C., Schreiber L., Bellazzini M., Ciliegi P., Assémat F., Baruffolo A., Biliotti V., Bregoli G., Cosentino G., D’Odorico S., Fusco T., Hubin N., Meimon S., Neichel B. Towards the phase A review of MAORY, the multiconjugate adaptive optics module for the E-ELT, this Symposium.

5. Diolaiti E., Conan J-M., Foppiani I., Lombini M., Petit C., Robert C., Schreiber L., Ciliegi P., Marchetti E., Bellazzini M., Busoni L., Esposito S., Fusco T., Hubin N., Quiros-Pacheco F., Baruffolo A., D’Odorico S., Farinato J., Neichel B., Ragazzoni R., Arcidiacono C., Biliotti V. , Bregoli G., Cosentino G., Innocenti G.,Proc. SPIE 7015, (2008), page 70150U-70150U-11

6. Ellerbroek, B. L., Rigaut F., J. Opt. Soc. Am. A Vol. 18, No. 10, (2001), page 2539-2547

7. Conan J-M., Fusco T., Neichel B., Petit C., Robert C., Wide Field AO design rules for ELTs, this Symposium.

8. R. Davies, AO Requirements of MICADO, the E-ELT imaging camera, this Symposium.

9. Boccas M., Vucina T., Araya C., Vera E., Ahhee C., Thin Solid Films 502, (2006),page 275-280 